\title{
Transplanted Neural Stem Cells: Playing a Neuroprotective Role by Ceruloplasmin in the Substantia Nigra of PD Model Rats?
}

\author{
Jia-Jia Xiao, ${ }^{1}$ Ming Yin, ${ }^{2}$ Ze-Jian Wang, ${ }^{2}$ and Xiao-Ping Wang ${ }^{1}$ \\ ${ }^{1}$ Department of Neurology, Shanghai First People's Hospital, School of Medicine, Shanghai Jiao Tong University, \\ Shanghai 200080, China \\ ${ }^{2}$ School of Pharmacy, Shanghai Jiao Tong University, Shanghai 200240, China
}

Correspondence should be addressed to Ze-Jian Wang; wangzejian@sjtu.edu.cn and Xiao-Ping Wang; wangxp@ustc.edu

Received 29 March 2015; Revised 15 May 2015; Accepted 17 May 2015

Academic Editor: Ryuichi Morishita

Copyright (C) 2015 Jia-Jia Xiao et al. This is an open access article distributed under the Creative Commons Attribution License, which permits unrestricted use, distribution, and reproduction in any medium, provided the original work is properly cited.

\begin{abstract}
Although mounting evidence suggests that ceruloplasmin (CP) deficiency and iron deposition are pivotal factors responsible for exacerbating demise of dopaminergic neurons in the substantia nigra (SN) of the Parkinsonism and neural stem cells (NSCs) are believed to be excellent candidates for compensating the lost dopaminergic neurons, there are few researches to explore the change of CP expression and of iron deposition in the pathological microenvironment of SN after NSCs transplantation and the ability of grafted NSCs to differentiate directionally into dopaminergic neurons under the changed homeostasis. With substantia nigral stereotaxic technique and NSCs transplantation, we found that tyrosine hydroxylase and CP expression decreased and iron deposition increased in the lesioned SN after 6-OHDA administration compared with control, while tyrosine hydroxylase and CP expression increased and iron deposition decreased after NSCs transplantation compared to 6-OHDA administration alone. Only a small number of embedding NSCs are able to differentiate into dopaminergic neurons. These results suggest that grafted NSCs have an influence on improving the content of CP expression, which may play a neuroprotective role by decreasing iron deposition and ameliorating damage of dopaminergic neurons and possibly underline the iron-related common mechanism of Parkinson's disease and Wilson's disease.
\end{abstract}

\section{Introduction}

Neural stem cells (NSCs) are renewable cells, famous for the specialties of self-replication and multilineage differentiation, making it possible to compensate the lost neurocytes and improve the symptoms of neurodegenerative diseases such as Parkinson's disease (PD) [1]. Exogenous transplantation is a promising strategy for rebuilding dopaminergic circuits and postponing process in PD [2]. In relevant animal models, NSCs transplantation has succeeded in neuroprotective effects against dopaminergic damage $[3,4]$. Previous studies reported that NSCs were able to migrate directly towards damaged substantia nigra $(\mathrm{SN})$ rather than other brain regions, disperse throughout the substantia nigra pars compacta, and develop partly neonatal dopaminergic neurons which are helpful to dopaminergic reinnervation [5-7]. The levels of gene expression of multifarious neural trophic factors such as nerve growth factor (NGF), brainderived neurotropic factor (BDNF), and neurotrophin-3
(NT-3) increase significantly in vitro NSCs culture and in vivo NSCs transplantation; these trophic factors can not only promote grafted NSCs to better adapt to ischemia microenvironment and facilitate homeostasis in brain, but also protect remaining endogenous brain cells against further damage $[8,9]$. NSCs proliferation and neural plasticity are seemingly associated with the progress of the disease and the severity of pathology $[10,11]$. However, it is more complicated for the pathogenesis and homeostasis in PD patients compared to animal models. There is a necessity to focus on the more efficient differentiation of NSCs towards dopaminergic neurons. But, at the same time, the changes of the pathologic microenvironment about the homeostasis of ceruloplasmin (CP) and iron should not be neglected, which may impact on NSCs function and PD symptoms after NSCs transplantation.

As a multicopper ferroxidase, $\mathrm{CP}$ is mainly expressed by astrocytes in the brain and anchored in the cytomembrane surface, involving iron metabolism and copper homeostasis both in PD and in Wilson's disease (WD) $[12,13]$. PD is 
considered to be closely related to the degraded ferroxidase activity and declined antioxidant defenses [14]. In the $\mathrm{SN}$, a deficiency of ferroxidase activity of $\mathrm{CP}$ contributes to the prooxidant ferrous iron accumulation and Parkinsonian neurodegeneration, resulting in 30\% nigral loss in CP knockout mice [15]. Aceruloplasminemia (aCP) [16] is characteristic with $\mathrm{CP}$ deficiency and iron deposition in central nervous system and peripheral tissue, causing diversified pathological alterations such as parkinsonism, craniofacial dyskinesia, cognitive impairment, and cerebellar ataxia, which is often present both in PD and in WD [10, 17]. Parkinsonism and iron concentration are attenuated by iron chelation with deferiprone in $\mathrm{CP}^{-/-}$mice and $\mathrm{aCP}[16,18]$. However, little information is available about the pathophysiological changes of iron and CP accompanying NSCs transplantation. Furthermore, the mechanism of NSCs differentiated into tyrosine hydroxylase-immunoreactive (TH-ir) neurons is still ambiguous [19]. Inconsistent results have been illustrated among different researches and this subject remains controversial $[11,20]$. The purpose of this paper is to evaluate the levels of iron, CP, and TH-ir neurons after NSCs transplantation in the SN of PD, which may offer theoretical support for replacement therapy with NSCs.

\section{Materials and Methods}

2.1. Animal Models. Female Sprague-Dawley rats (Slac Laboratory Animal Technology Co., Ltd.) weighing 180-220 g were housed under the circumstance of room temperature, constant airflow, acoustic isolation, and $12 \mathrm{~h}$ light/dark cycles, with ad libitum food and water. All the animals were randomly split into three groups: control, 6-OHDAtreated, and NSC-grafted 6-OHDA-treated groups. Rats in 6-OHDA-treated and NSC-grafted 6-OHDA-treated groups were anesthetized with chloral hydrate $(400 \mathrm{mg} / \mathrm{kg}$, i.p. $)$ and immobilized to the stereotaxic apparatus. A small burr hole was drilled into the skull above the right medial forebrain bundle (MFB) region and then 6-OHDA $(2 \mu \mathrm{g} / \mu \mathrm{L}$, Sigma) was microinjected $(8 \mu \mathrm{L})$ at the rate of $0.5 \mu \mathrm{L} / \mathrm{min}$ into the right MFB using stereotaxic technique in accordance with two coordinates: anteroposterior (AP): $-1.8 \mathrm{~mm}$, mediolateral (ML): $2.5 \mathrm{~mm}$, and dorsoventral (DV): $-7.5 \mathrm{~mm}$ and AP: $-1.8 \mathrm{~mm}$, ML: $2.5 \mathrm{~mm}$, and DV: $-8.0 \mathrm{~mm}$ from bregma [21-23]. After microinjection, the needle was left in coordinate site for $5 \mathrm{~min}$ before being removed slowly. Healthy rats without any process were selected as control. All the rats received rotational tests by apomorphine hydrochloride $(0.05 \mathrm{mg} / \mathrm{kg}$, s.c., Sigma) one week before and four weeks after surgery. Asymmetrical rotations were counted within $30 \mathrm{~min}$ and reached more than 7 rotations/min; they were deemed to be PD model rats [24]. Each group was of fifteen rats: five rats were used for immunohistochemical staining, five for Western blot, and also five for real-time PCR. All researches were conducted strictly according to the Guide for the Care and Use of Laboratory Animals (NIH) and approved by the Animal Ethics Committee of Shanghai Jiao Tong University.
2.2. NSCs Transplantation. NSCs were acquired from the subventricular zone (SVZ) of E14 fetal rat brains. The dissociated tissue was digested with $0.25 \%$ Trypsin-EDTA and the cell suspension was then incubated in serum-free medium (DMEM/F12, Hyclone) containing epidermal growth factor (EGF, $20 \mathrm{ng} / \mathrm{mL}$, Invitrogen), basic fibroblast growth factor (bFGF, $20 \mathrm{ng} / \mathrm{mL}$, Invitrogen), and B27 supplement ( $20 \mu \mathrm{L} / \mathrm{mL}$, Invitrogen). Four weeks after 6-OHDA lesion, PD rats were injected $5 \mu \mathrm{L}$ NSCs suspension $\left(5 \times 10^{4}\right.$ cells $\left./ \mu \mathrm{L}\right)$ into the right $\mathrm{SN}$ as the following coordinate: AP: $-5.2 \mathrm{~mm}$, ML: $1.8 \mathrm{~mm}$, and DV: $-7.8 \mathrm{~mm}$ [6]. The speed was $0.5 \mu \mathrm{L} / \mathrm{min}$ and needle retaining time was $10 \mathrm{~min}$.

2.3. Histological Studies. All the animals were killed over eight weeks after the last treatment. Rats were fully anesthetized and perfused transcardially with $200 \mathrm{~mL}$ of cold PBS, followed by $300 \mathrm{~mL} 4 \%$ paraformaldehyde. The harvested brains were immersed in the $4 \%$ paraformaldehyde $\left(4^{\circ} \mathrm{C}\right)$ for fixation and subsequently soaked in $20 \%$ and $30 \%$ sucrose solutions $\left(4^{\circ} \mathrm{C}\right)$ for dehydration. The coronal sections of $\mathrm{SN}$ were cut into $10 \mu \mathrm{m}$ on a freezing microtome and stored at $-20^{\circ} \mathrm{C}$.

2.4. Iron Staining. Iron staining was on the basis of Perls' Prussian blue reaction: the sections were washed for three times with $0.01 \mathrm{M}$ phosphate-buffered saline (PBS) and incubated for $30 \mathrm{~min}$ at $37^{\circ} \mathrm{C}$ in the mixed solution of equal parts, $2 \% \mathrm{HCl}$ and $2 \%$ potassium ferrocyanide freshly prepared. By washing three times with PBS, sections were soaked in $99.7 \%$ methanol containing $0.3 \%$ hydrogen peroxide for $20 \mathrm{~min}$ in order to inactivate endogenous peroxidases vitality. They were washed again and then coloured in the solution of diaminobenzidine (DAB) for $10 \mathrm{~min}$.

2.5. Immunohistochemistry. The sections were washed three times by $0.01 \mathrm{M} \mathrm{PBS}$ and treated with $0.3 \%$ hydrogen peroxide methanol solution for $20 \mathrm{~min}$ to suppress endogenous peroxidases activity. After washing thrice in PBS, these sections were treated for $30 \mathrm{~min}$ at room temperature with $5 \%$ bovine serum albumin for the purpose of reducing nonspecific binding and then incubated overnight with rabbit anti-rat TH $(1: 1,000$, GeneTex) [27] and rabbit anti-rat CP $(1: 1,000$, Epitomics) [28] at $4^{\circ} \mathrm{C}$ in a humidified chamber. The sections were washed with PBS and incubated with biotinylated goat anti-rabbit IgG (Boster) for $30 \mathrm{~min}$ at $37^{\circ} \mathrm{C}$, accompanied with enlargement through streptavidin peroxidase for $30 \mathrm{~min}$ at the same temperature. Afterwards, sections were rinsed by PBS and coloured with DAB kits for $10 \mathrm{~min}$.

2.6. Neuronal Counting. Sections were counted manually by a light microscopy with $\times 400$ magnification and three images per section were taken to calculate positive neurons at an average. The counting areas were mainly located in the central substantia nigra pars compacta (SNpc) region. All counts and assessments were carried out blindly to the treatments received. 


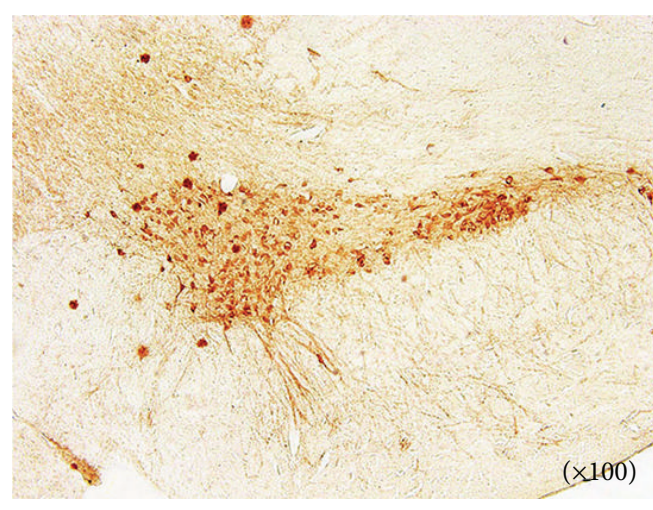

(a)

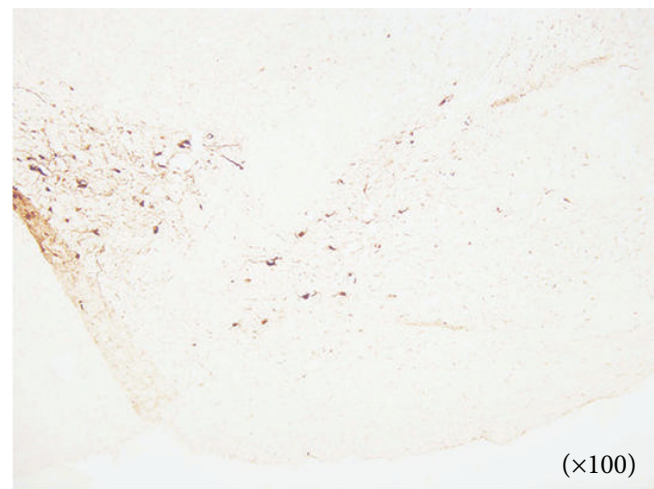

(c)

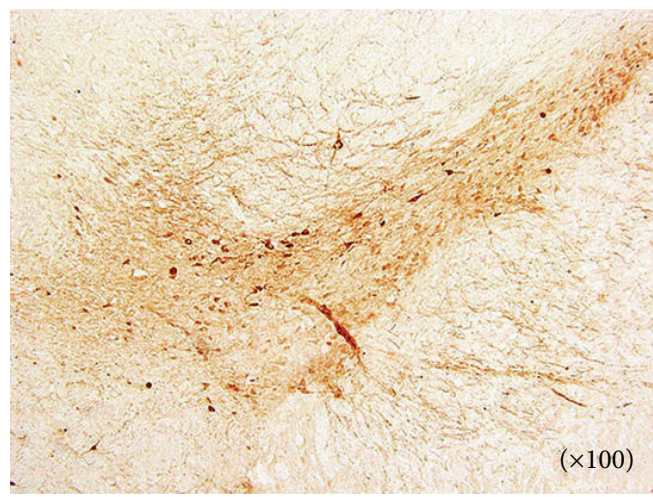

(e)

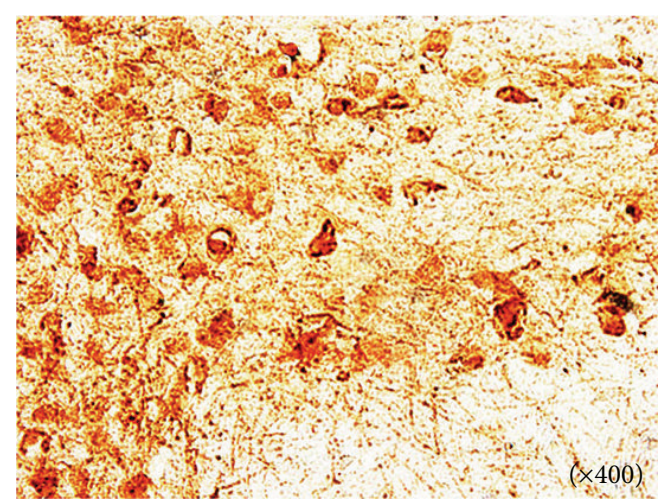

(b)

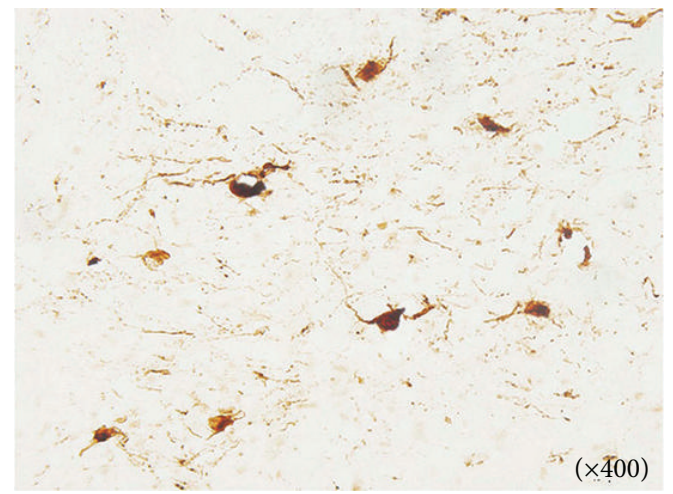

(d)

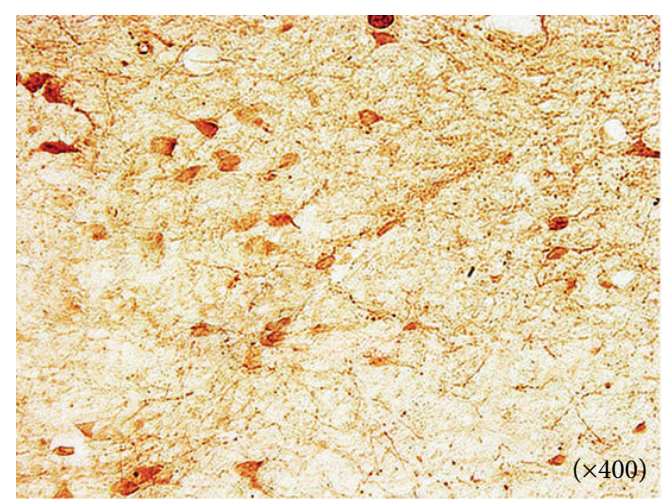

(f)

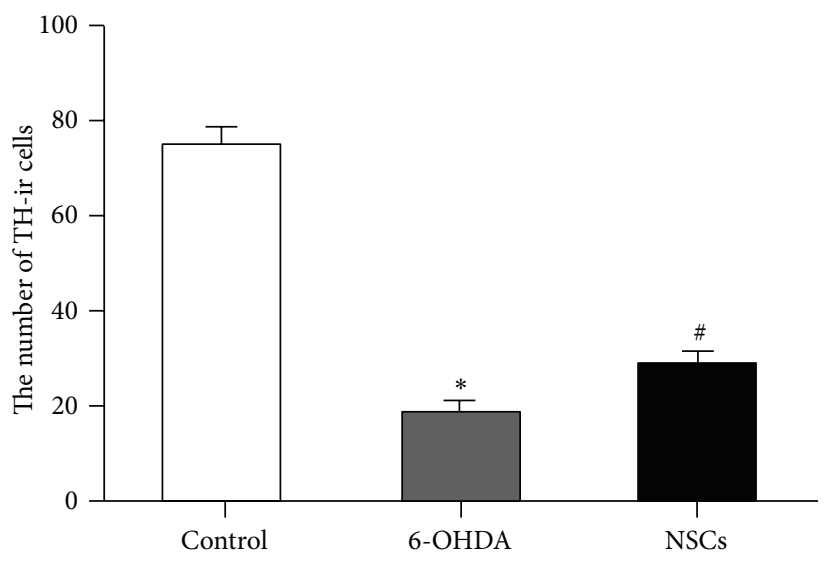

(g)

Figure 1: Continued. 

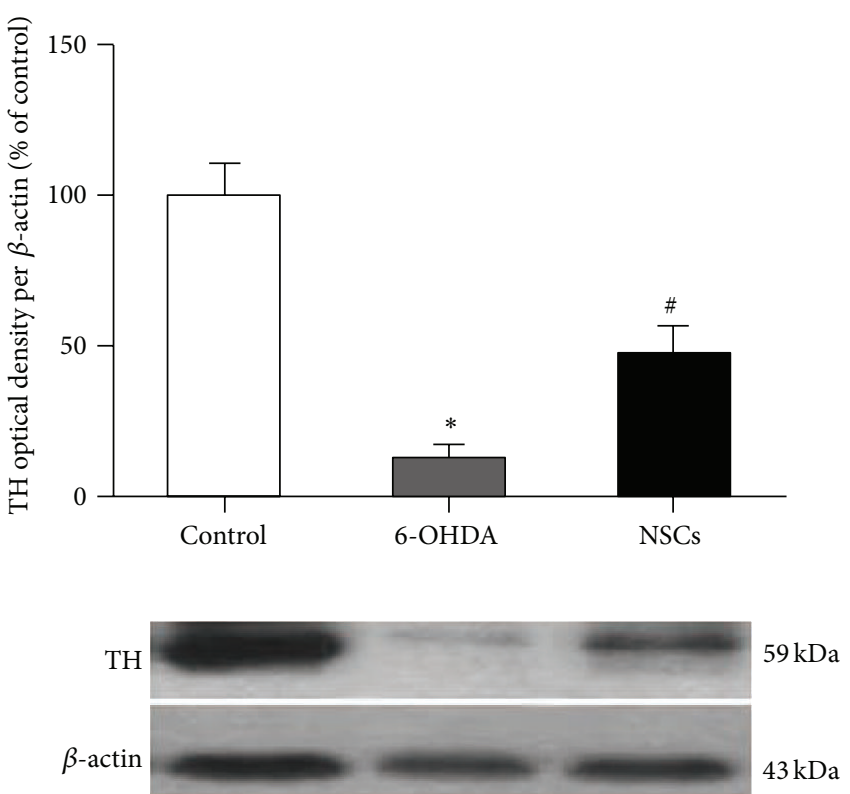

(h)

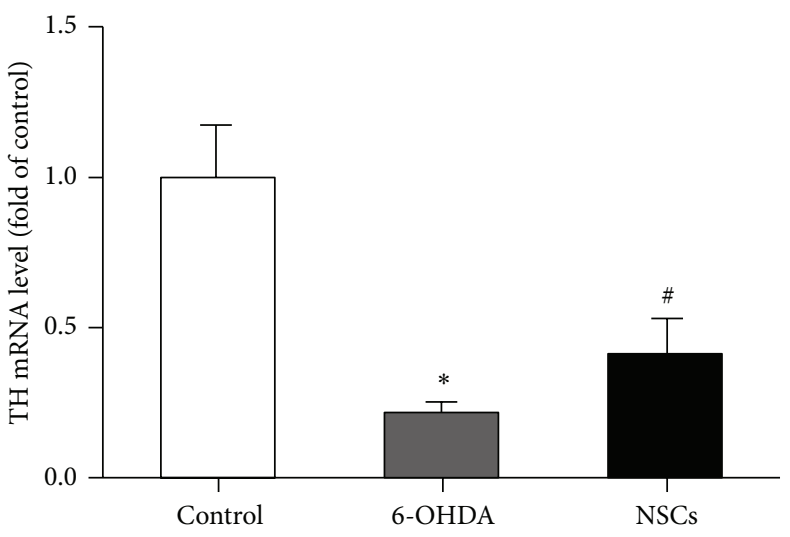

(i)

FIgURE 1: Influences of grafted NSCs on the TH-ir neurons in the SN of 6-OHDA-induced PD rats. (a, b) TH-ir neurons in control rats; (c, d) TH-ir cells in 6-OHDA-treated rats; (e, f) TH-ir neurons in NSCs-treated rats; (g, h, and i) the average total number of TH-ir neurons, Western blot, and real-time quantitative PCR in different groups: TH-ir neurons significantly decreased in the SN of 6-OHDA-treated rats compared with control rats $\left({ }^{*} p<0.05\right)$, while they increased in NSCs-treated rats compared with 6-OHDA-treated rats $\left({ }^{\#} p<0.05\right)$.

2.7. Western Blot. Fresh right nigral tissues were dissected out, respectively, from 6-OHDA-treated, NSC-grafted 6OHDA-treated and control groups, homogenized in cold lysis buffer supplemented with $1 \mathrm{M}$ Tris- $\mathrm{HCl}, 1 \%$ nonidet $\mathrm{p}$ $40,1 \%$ Triton X-100, $0.5 \%$ sodium deoxycholate, $10 \%$ SDS, 0.5 M EDTA, 1 mM PMSF, and protease inhibitor cocktail $(10 \mu \mathrm{g} / \mathrm{mL}$ aprotinin, $10 \mu \mathrm{g} / \mathrm{mL}$ leupeptin), and then centrifuged at $12000 \times \mathrm{g}$ for $10 \mathrm{~min}$ at $4^{\circ} \mathrm{C}$ to collect the supernatant. Protein concentrations were assessed by the Bradford protein assay. Equal amounts of protein were diluted in loading buffer and then separated by SDS-PAGE (10\% gradient gels) and transferred to nitrocellulose membrane (CP: $120 \mathrm{~V}$, $120 \mathrm{~min}$; TH: $60 \mathrm{~V}, 90 \mathrm{~min}$ ). After transfer, samples were washed three times with TBST for 5 min per time and blocked by $5 \%$ skim milk-TBST buffer for $2 \mathrm{~h}$ at room temperature. The membranes were incubated at $4^{\circ} \mathrm{C}$ overnight with primary antibodies, anti-CP (1:2000, Epitomics) and anti-TH (1:2000, GeneTex), and then incubated with the secondary antibody (1:20000, Boster) for 1 hour at room temperature. Target proteins were visualized by using enhanced chemiluminescence reagents. Densitometry analysis was applied to assess semiquantifications of the signals with the MetaMorph software.

2.8. Real-Time Quantitative PCR. Total RNA was purified by homogenization in Trizol reagent from right nigral tissues. Extracted RNA $(2 \mu \mathrm{g})$ was transcribed reversely into cDNA using the AMV reverse transcription system. The primer sequences were shown in Table 1.

PCR amplifications were carried out as the following conditions: step 1, $5 \mathrm{~min}$ at $94^{\circ} \mathrm{C}$ for one cycle and step 2, $30 \mathrm{~s}$
TABLE 1

\begin{tabular}{ll}
\hline Gene & Sequence $\left(5^{\prime}-3^{\prime}\right)$ \\
\hline CP [25] & $\begin{array}{l}\text { Forward: GTA TGT GAT GGC TAT GGG CAA TGA } \\
\text { Reverse: CCT GGA TGG AAC TGG TGA TGG A }\end{array}$ \\
\hline \multirow{2}{*}{ TH [26] } & $\begin{array}{l}\text { Forward: AGC TGT GCA GCC CTA CCA AGA } \\
\text { Reverse: GTG TGT ACG GGT CAA ACT TCA CAG A }\end{array}$ \\
\hline \multirow{2}{*}{-actin } & $\begin{array}{l}\text { Forward: CCT CTA TGC CAA CAC AGT } \\
\text { Reverse: AGC CAC CAA TCC ACA CAG }\end{array}$ \\
\hline
\end{tabular}

at $94^{\circ} \mathrm{C}, 30 \mathrm{~s}$ at $57^{\circ} \mathrm{C}$, and $1 \mathrm{~min}$ at $72^{\circ} \mathrm{C}$, for 30 cycles [25]. The data were assessed by the delta-delta Ct method $\left(2^{-\Delta \Delta \mathrm{CT}}\right)$.

2.9. Statistical Analysis. SPSS 19.0 software was used to statistically analyze all data. The results were expressed exactly as the mean \pm SD and assessed by one-way ANOVA followed by the SNK test. Probability $p$ value of less than 0.05 was deemed to be statistically significant difference.

\section{Results}

3.1. Grafted NSCs Increased TH-ir Neurons in the SN of 6OHDA-Induced PD Rats. TH-ir neurons presented multiple dendrites, plump somata, and high density in the SN of normal rats (75.42 \pm 11.24 ) (Figures $1(\mathrm{a})$ and $1(\mathrm{~b})$ ), while there were few dendrites, slight somata, and significantly decreased density of TH-ir neurons in the lesioned SN of 6-OHDAtreated $(18.75 \pm 8.98)$ (Figures $1(\mathrm{c})$ and $1(\mathrm{~d})$ ) and NSCgrafted 6-OHDA-treated (28.58 \pm 9.17) (Figures 1(e) and 1(f)) 


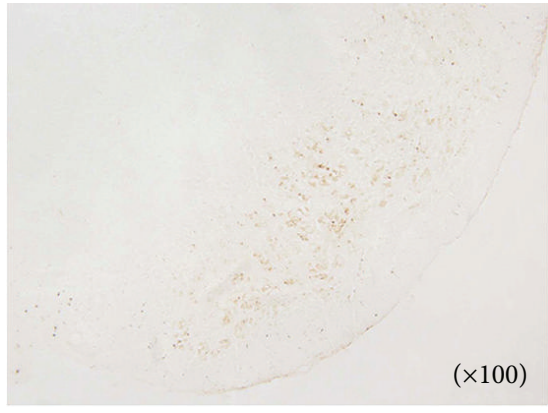

(a)

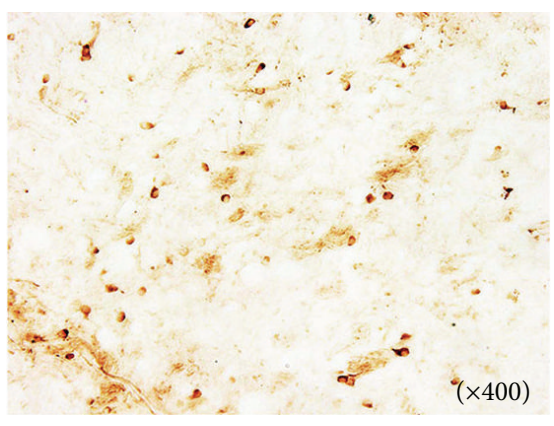

(d)

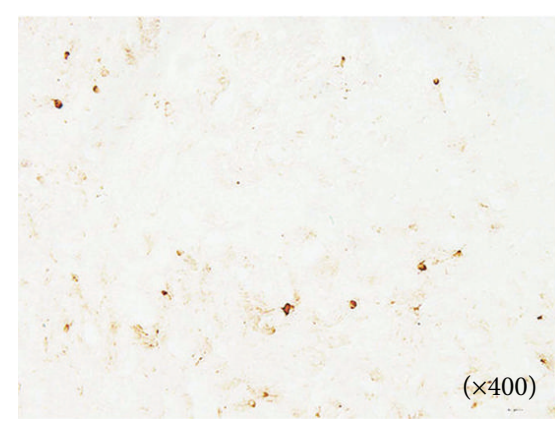

(b)

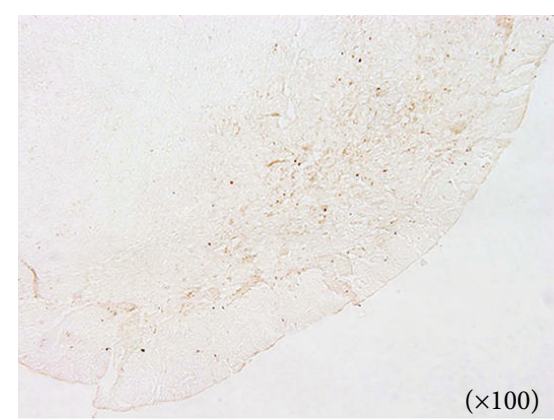

(e)

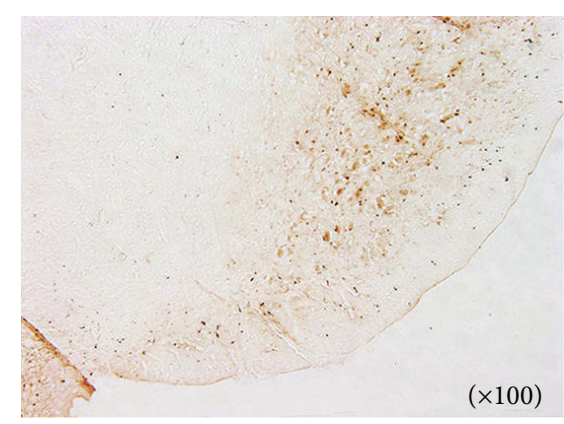

(c)

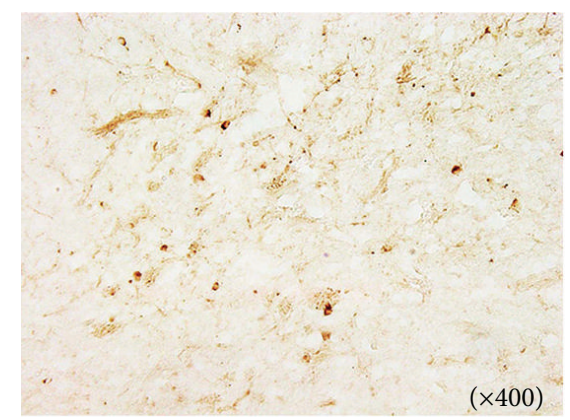

(f)

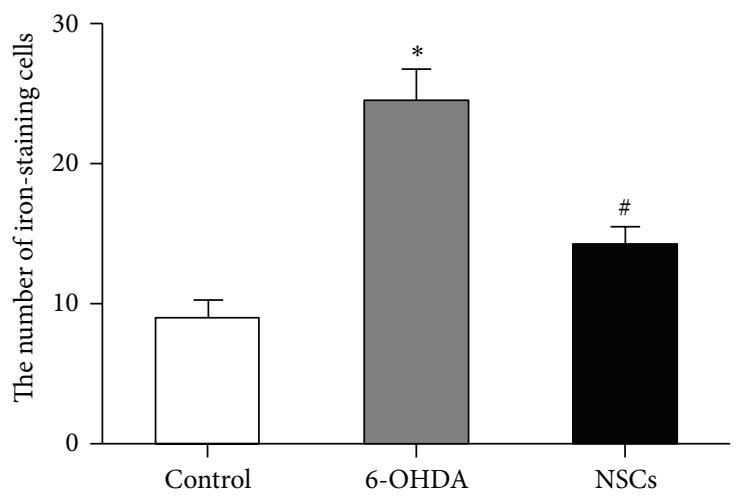

(g)

FIGURE 2: Effects of transplanted NSCs on the iron-staining cells in the SN of 6-OHDA-induced PD rats. (a, b) Iron-staining cells in control rats; $(c, d)$ iron-staining cells in 6-OHDA-treated rats; $(e, f)$ iron-staining cells in NSCs-treated rats; $(g)$ the average total number of ironstaining cells in different groups: iron-staining cells dramatically increased in the SN of 6-OHDA-treated rats compared with control rats $\left({ }^{*} p<0.05\right)$, while they decreased in NSCs-treated rats compared with 6-OHDA-treated rats $\left({ }^{\#} p<0.05\right)$.

groups. Cell counting (Figure 1(g)) indicated that the number of $\mathrm{TH}$-ir neurons was, respectively, decreased by $75 \%$ and $62 \%$. Compared with the 6-OHDA-treated rats, the density of TH-ir neurons in NSC-grafted 6-OHDA-treated groups was increased by $52 \%$ after grafting NSCs but still far from normal level. NSCs treatment induced a mild increase in TH mRNA expression and protein synthesis in lesioned SN (Figures $1(\mathrm{~h})$ and $1(\mathrm{i})$ ).

3.2. Transplanted NSCs Decreased Iron-Staining Cells in the SN of 6-OHDA-Induced PD Rats. In the SN of healthy rats, a very small quantity of iron-staining cells $(8.83 \pm 5.13)$ (Figures 2(a) and 2(b)) was observed. But in the lesioned SN of 6OHDA-treated groups, the positive number of iron-staining cells was dramatically increased by $178 \%(24.54 \pm 7.86)$ (Figures 2(c) and 2(d)); the staining density of the lesioned to the healthy side was obviously darkened and turbid. After transplanting NSCs, the positive cells amount was decreased by $42 \%(14.17 \pm 4.75)$ (Figures $2(\mathrm{e})$ and $2(\mathrm{f})$ ).

\subsection{Embedding NSCs Enhanced CP Expression in the SN of} 6-OHDA-Induced PD Rats. There were massive CP-immunoreactive (CP-ir) cells $(79.83 \pm 12.19)$ (Figures $3(a)$ and $3(b))$ in the physiological microenvironment of $\mathrm{SN}$ in control group. However, the quantity of CP-ir cells in surgery groups was diminished especially in 6-OHDA-treated groups. Staining results showed that the density of CP-ir cells in 


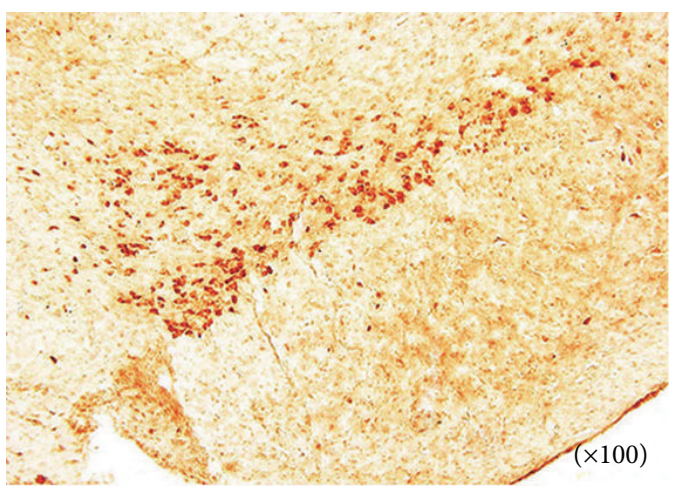

(a)

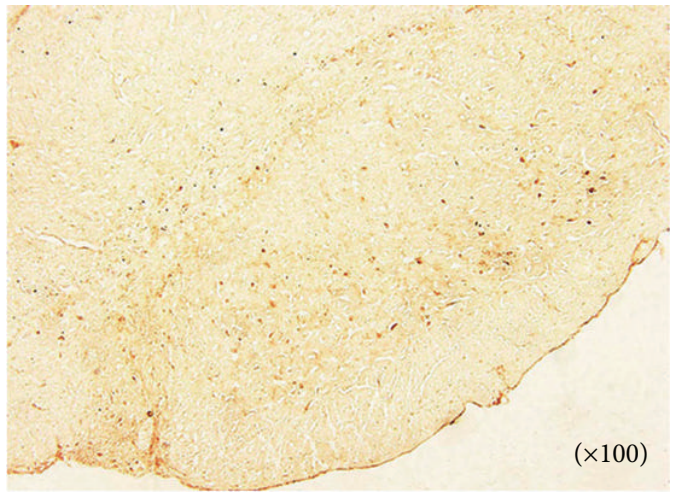

(c)

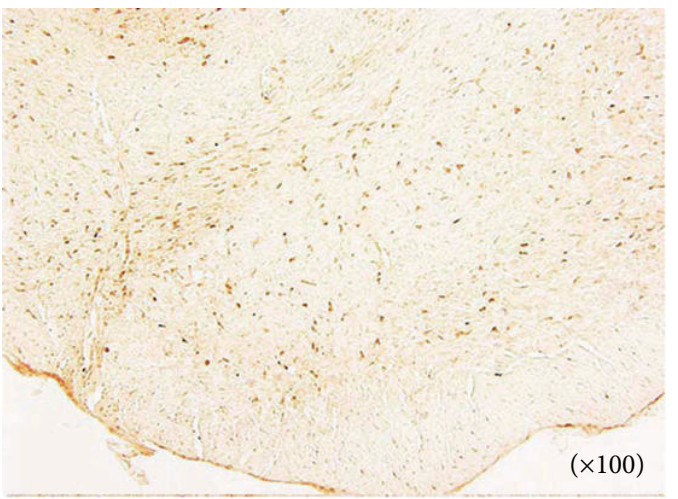

(e)

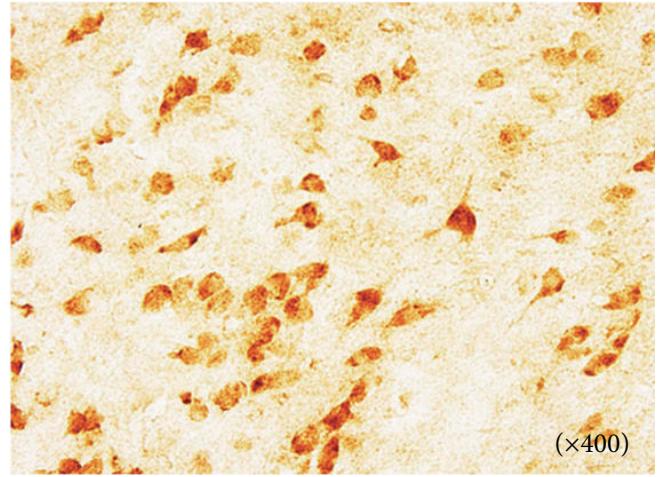

(b)

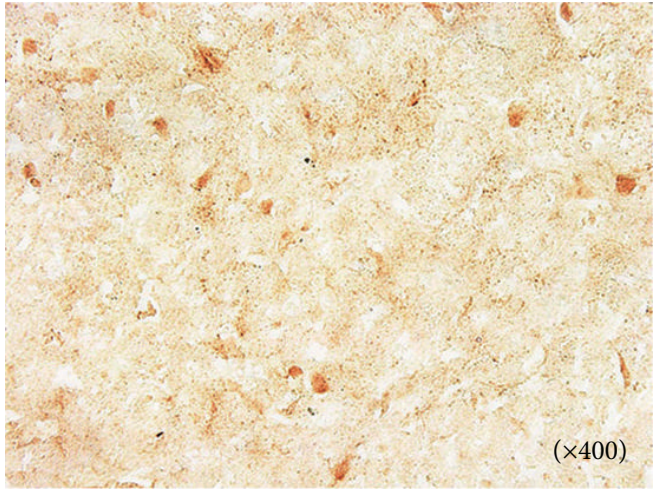

(d)

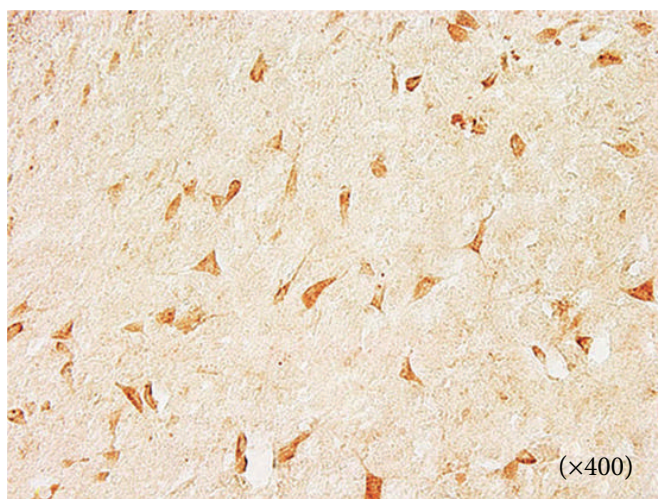

(f)

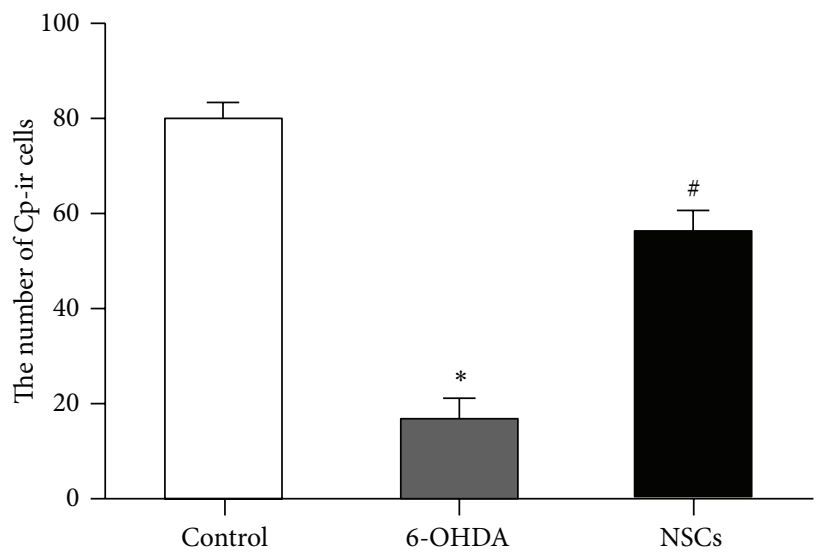

(g)

Figure 3: Continued. 


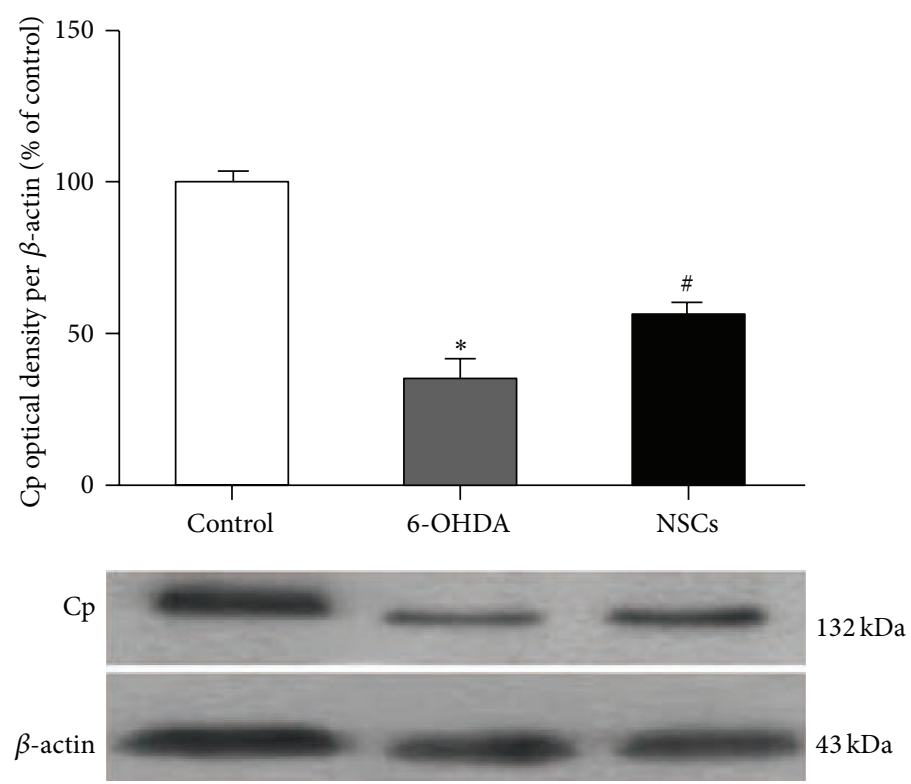

(h)

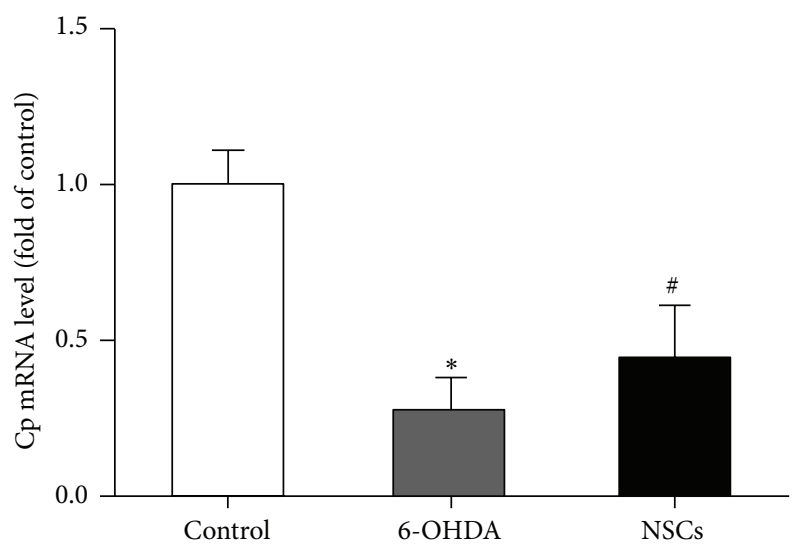

(i)

Figure 3: Impacts of exogenous NSCs on the Cp-ir cells in the SN of 6-OHDA-induced PD rats. (a, b) Cp-ir cells in control rats; (c, d) Cp-ir cells in 6-OHDA-treated rats; (e, f) Cp-ir cells in NSCs-treated rats; (g, h, and i) the average total number of Cp-ir cells, Western blot, and real-time quantitative PCR in different groups: Cp-ir cells significantly decreased in the SN of 6-OHDA-treated rats compared with control rats $\left({ }^{*} p<0.05\right)$, while they increased in NSCs-treated rats compared with 6-OHDA-treated rats $\left({ }^{*} p<0.05\right)$.

the lesioned SN of 6-OHDA-treated and NSC-grafted 6OHDA-treated groups was, respectively, decreased by $78 \%$ $(17.25 \pm 11.34)$ (Figures 3(c) and $3(\mathrm{~d}))$ and 30\% (56.13 \pm 13.20) (Figures 3(e) and 3(f)) compared to control groups. Notably, exogenous NSCs enhanced CP expression in mRNA and protein level (Figures 3(h) and 3(i)) and also increased CP-ir cells (Figure 3(g)).

\section{Discussion}

TH-ir neurons are well-suited for assessing the loss of dopaminergic cells as well as the differentiation of NSCs in PD. In our study, grafted NSCs have also exerted protection on the remaining dopaminergic neuron and impact on proliferation in the 6-OHDA-induced rats. After injecting NSCs, the number of TH-ir neurons increased slightly but did not reach the physiological amounts. As was reported, more than $50 \%$ of the newly generated neurons will confront apoptosis and approximately $5-10 \%$ of the grafted NSCs expressed TH-ir neurons $[29,30]$. Numerous lines of evidence have demonstrated that $\mathrm{TH}$-ir neurons are significantly lessened in the SN of Parkinson's rats $[15,31]$ and exogenous NSCs transplanted into $\mathrm{SN}$ are able to survive, migrate, and also differentiate [11]. Nevertheless, in the midbrain of PD, the low oxygen tension, through upregulating hypoxia inducible factor-1 (HIF-1), accelerates NSCs proliferation, stimulates NSCs that differentiate into neonatal dopaminergic neurons which have been verified to secrete dopamine, and promotes erythropoietin (EPO) and vascular endothelial growth factor (VEGF) expression to further exert neuroprotective effects on midbrain dopaminergic neurons [32]. Moreover, reactive astrocytes support $\mathrm{TH}$-ir neurons development and survival [33], and transplanted NSCs tend to differentiate into more astrocytes than dopaminergic neurons in our study. Interestingly, undifferentiated neural progenitor cells (NPCs) from mouse embryos embedded into the lesioned mouse striatum preferentially differentiate into neurons but not glial cells [34]. Perhaps such discrepancies are caused by various origins of grafted cells or different conditions of cell culture.

As a high activity of electron transfer, ferrous iron is supposed to be a forceful catalyst for the production of reactive oxygen species (ROS) by Fenton reaction; its accumulation will injure dopaminergic cytomembrane and chondriosome; as a result, dopaminergic neurons are degenerated in a large amount in PD [35]. Iron-staining cells are the damaged neurons and gliacytes, but dopaminergic neurons are more vulnerable. Supporting this notion is the fact that SN in the brain is the second highest concentration of iron behind globus pallidus [36], in which iron progressively deposits with aging process [37], coincident with areas more susceptible to insults. The oxidases such as glutathione peroxidase (GSH) and superoxide dismutase (SOD) have a considerable shortage in dopaminergic neurons of SN compared to other regions of the brain [38, 39]. The lack of ferritin and neuromelanin digested by microglia in the dopaminergic neurons leads to the fact that intracellular iron exists mainly in the form of free ferrous iron [40]. What is more, high amounts of iron released from damaged neurogliocytes such as astrocytes and microglia promote dopaminergic neurons uptake [41]. Analogously, iron also accumulates in basal ganglia occurring in the whole pathological process of WD and induces secondary damage to affected tissues, although 
the deposition of copper has been verified to be the main pathogenic factors [42]. In the $\mathrm{SN}$ of Parkinson's rats, there are fewer TH-ir neurons but more iron-staining cells. In our study, we further observed that the density of ironstaining cells declined slightly in the lesioned SN after NSCs transplantation, suggesting that grafted NSCs might relieve iron deposition in dopaminergic neurons by enhancing CP content and activity.

Our study also showed that CP levels in the SN of NSCgrafted 6-OHDA-treated $\mathrm{PD}$ rats were predominantly higher than PD rats. Intracellular iron decrease was concurrent with increased levels of CP mRNA and protein. These phenomena suggest that transplanted NSCs are very likely to play a neuroprotective role by increasing the expression level of $\mathrm{Cp}$, which may decline the deposition of intracellular iron by increasing the expression of ferroportin in the membrane surface of dopaminergic neurons or by decreasing ferrous iron into the cells. The synthesis of $\mathrm{CP}$ in the $\mathrm{SN}$ plays a crucial role in eliminating ferrous iron and protecting against ironmediated free-radical injury towards TH-ir neurons [43]. High activity of $\mathrm{CP}$ promotes stable expression of ferroportin (Fpn), by which excessive iron will be exported from neuroglia cells and dopaminergic neurons to extracellular matrix [44]. Once the content and activity of CP decline, due to gene mutation, aging process, or toxic tort, ferrous iron will access cytomembrane conveniently by means of divalent metal transporter (DMT1). Similarly, CP-deficient mice are more vulnerable in oxidative stress rather than wildtype mice [45]. Besides, Fpn expressing in low level leads to decreased iron efflux, all of which aggravate cellular iron overload and oxidative damage [46].

In conclusion, NSCs are capable of differentiating into TH-ir neurons and ameliorating surrounding microenvironment in the SN of Parkinson's rats. But most of all, by increasing the number of $\mathrm{CP}$-ir cells and improving the content of CP, exogenous NSCs transplantation declines the concentration of iron followed by relieving damage of dopaminergic neurons and may ameliorate the iron-related oxidative injuries of PD and WD.

\section{Conflict of Interests}

The authors declare that there is no conflict of interests regarding the publication of this paper.

\section{Acknowledgment}

This study was supported by Grants from the National Natural Science Foundation of China (81071065).

\section{References}

[1] X. Tan, L. Zhang, H. Zhu et al., "Brn4 and TH synergistically promote the differentiation of neural stem cells into dopaminergic neurons," Neuroscience Letters, vol. 571, pp. 23-28, 2014.

[2] R. Pardal and J. López-Barneo, "Neural stem cells and transplantation studies in Parkinson's disease," Advances in Experimental Medicine and Biology, vol. 741, pp. 206-216, 2012.
[3] T. Yasuhara, N. Matsukawa, K. Hara et al., "Transplantation of human neural stem cells exerts neuroprotection in a rat model of Parkinson's disease," The Journal of Neuroscience, vol. 26, no. 48, pp. 12497-12511, 2006.

[4] K. B. Bjugstad, Y. D. Teng, D. E. Redmond Jr. et al., "Human neural stem cells migrate along the nigrostriatal pathway in a primate model of Parkinson's disease," Experimental Neurology, vol. 211, no. 2, pp. 362-369, 2008.

[5] K. K. Meissner, D. L. Kirkham, and L. C. Doering, “Transplants of neurosphere cell suspensions from aged mice are functional in the mouse model of Parkinson's," Brain Research, vol. 1057, no. 1-2, pp. 105-112, 2005.

[6] Q. F. Zhu, J. Ma, L. L. Yu, and C. G. Yuan, "Grafted neural stem cells migrate to substantia nigra and improve behavior in Parkinsonian rats," Neuroscience Letters, vol. 462, no. 3, pp. 213218, 2009.

[7] K. B. Bjugstad, D. E. Redmond Jr., Y. D. Teng et al., "Neural stem cells implanted into MPTP-treated monkeys increase the size of endogenous tyrosine hydroxylase-positive cells found in the striatum: a return to control measures," Cell Transplantation, vol. 14, no. 4, pp. 183-192, 2005.

[8] D. M. Hermann, L. Peruzzotti-Jametti, J. Schlechter, J. D. Bernstock, T. R. Doeppner, and S. Pluchino, "Neural precursor cells in the ischemic brain-integration, cellular crosstalk, and consequences for stroke recovery," Frontiers in Cellular Neuroscience, vol. 8, article 291, 2014.

[9] C. Sun, H. Zhang, J. Li et al., "Modulation of the major histocompatibility complex by neural stem cell-derived neurotrophic factors used for regenerative therapy in a rat model of stroke," Journal of Translational Medicine, vol. 8, article 77, 2010.

[10] K. J. Doorn, B. Drukarch, A. van Dam, and P. J. Lucassen, "Hippocampal proliferation is increased in presymptomatic Parkinson's disease and due to microglia," Neural Plasticity, vol. 2014, Article ID 959154, 13 pages, 2014.

[11] Z. H. Sun, Y. L. Lai, W. W. Zeng, D. Zhao, H. C. Zuo, and Z. P. Xie, "Neural stem/progenitor cells survive and differentiate better in PD rats than in normal rats," Acta Neurochirurgica Supplement, vol. 87, pp. 169-174, 2003.

[12] V. Vassiliev, Z. L. Harris, and P. Zatta, "Ceruloplasmin in neurodegenerative diseases," Brain Research Reviews, vol. 49, no. 3, pp. 633-640, 2005.

[13] S. Olivieri, A. Conti, S. Iannaccone et al., "Ceruloplasmin oxidation, a feature of Parkinson's disease CSF, inhibits ferroxidase activity and promotes cellular iron retention," Journal of Neuroscience, vol. 31, no. 50, pp. 18568-18577, 2011.

[14] M. Rubio-Osornio, S. Montes, Y. Heras-Romero et al., "Induction of ferroxidase enzymatic activity by copper reduces $\mathrm{MPP}^{+}$evoked neurotoxicity in rats," Neuroscience Research, vol. 75, no. 3, pp. 250-255, 2013.

[15] S. Ayton, P. Lei, P. A. Adlard et al., "Iron accumulation confers neurotoxicity to a vulnerable population of nigral neurons: implications for Parkinson's disease," Molecular Neurodegeneration, vol. 9, no. 1, article 27, 2014.

[16] A. McNeill, M. Pandolfo, J. Kuhn, H. Shang, and H. Miyajima, "The neurological presentation of ceruloplasmin gene mutations," European Neurology, vol. 60, no. 4, pp. 200-205, 2008.

[17] W.-J. Li, J.-F. Wang, and X.-P. Wang, "Wilson's disease: update on integrated Chinese and western medicine," Chinese Journal of Integrative Medicine, vol. 19, no. 3, pp. 233-240, 2013.

[18] S. Ayton, P. Lei, J. A. Duce et al., "Ceruloplasmin dysfunction and therapeutic potential for Parkinson disease," Annals of Neurology, vol. 73, no. 4, pp. 554-559, 2013. 
[19] Y. Yu and T. Wen, "Generation of dopaminergic neurons by fusion of neural stem cells and midbrain neurons," Cellular and Molecular Biology, vol. 57, supplement, pp. OL1528-OL1533, 2011.

[20] X. Deng, Y. Liang, H. Lu et al., "Co-transplantation of GDNFoverexpressing neural stem cells and fetal dopaminergic neurons mitigates motor symptoms in a rat model of parkinson's disease," PLoS ONE, vol. 8, no. 12, Article ID e80880, 2013.

[21] G. Paxinos and C. Watson, The Rat Brain Stereotaxic Coordinates, Academic Press, Sydney, Australia, 2006.

[22] L. Frau, M. Morelli, and N. Simola, "Performance of movement in hemiparkinsonian rats influences the modifications induced by dopamine agonists in striatal efferent dynorphinergic neurons," Experimental Neurology, vol. 247, pp. 663-672, 2013.

[23] X.-X. Du, H.-M. Xu, H. Jiang, N. Song, J. Wang, and J.-X. $\mathrm{Xie}$, "Curcumin protects nigral dopaminergic neurons by ironchelation in the 6-hydroxydopamine rat model of Parkinson's disease," Neuroscience Bulletin, vol. 28, no. 3, pp. 253-258, 2012.

[24] J. Wang, H. Xu, H. Jiang, X. Du, P. Sun, and J. Xie, "Neurorescue effect of rosmarinic acid on 6-hydroxydopamine-lesioned nigral dopamine neurons in rat model of Parkinson's disease," Journal of Molecular Neuroscience, vol. 47, no. 1, pp. 113-119, 2012.

[25] L. Li, C. Holscher, B.-B. Chen, Z.-F. Zhang, and Y.-Z. Liu, "Hepcidin treatment modulates the expression of divalent metal transporter-1, ceruloplasmin, and ferroportin-1 in the rat cerebral cortex and hippocampus," Biological Trace Element Research, vol. 143, no. 3, pp. 1581-1593, 2011.

[26] Y. Li, Y. Sun, J. Yang, Y. Wu, J. Yu, and B. Li, “Age-dependent dopaminergic dysfunction following fetal exposure to atrazine in SD rats," Environmental Toxicology and Pharmacology, vol. 37, no. 3, pp. 1275-1282, 2014.

[27] J. Y. Lee, S. H. Kim, A.-R. Ko et al., "Therapeutic effects of repetitive transcranial magnetic stimulation in an animal model of Parkinson's disease," Brain Research, vol. 1537, pp. 290-302, 2013.

[28] R. K. Leak, K. A. Garbett, A. M. Dettmer, Z. Zhang, K. Mirnics, and J. L. Cameron, "Physical activity is linked to ceruloplasmin in the striatum of intact but not MPTP-treated primates," Cell and Tissue Research, vol. 350, no. 3, pp. 401-407, 2012.

[29] M. Biebl, B. Winner, and J. Winkler, "Caspase inhibition decreases cell death in regions of adult neurogenesis," NeuroReport, vol. 16, no. 11, pp. 1147-1150, 2005.

[30] J. Ourednik, V. Ourednik, W. P. Lynch, M. Schachner, and E. Y. Snyder, "Neural stem cells display an inherent mechanism for rescuing dysfunctional neurons," Nature Biotechnology, vol. 20, no. 11, pp. 1103-1110, 2002.

[31] X. Zhang, J. Xiong, S. Liu et al., "Puerarin protects dopaminergic neurons in Parkinson's disease models," Neuroscience, vol. 280, pp. 88-98, 2014.

[32] C. Krabbe, S. T. Bak, P. Jensen et al., "Influence of oxygen tension on dopaminergic differentiation of human fetal stem cells of midbrain and forebrain origin," PLOS ONE, vol. 9, no. 5, Article ID e96465, 2014.

[33] X.-L. Gu, C.-X. Long, L. Sun, C. Xie, X. Lin, and H. Cai, "Astrocytic expression of Parkinson's disease-related A53T-synuclein causes neurodegeneration in mice," Molecular Brain, vol. 3, no. 1, article 12, 2010.

[34] D. Ziavra, G. Makri, P. Giompres et al., "Neural stem cells transplanted in a mouse model of Parkinson's disease differentiate to neuronal phenotypes and reduce rotational deficit," CNS and Neurological Disorders_Drug Targets, vol. 11, no. 7, pp. 829-835, 2012.
[35] A. Wypijewska, J. Galazka-Friedman, E. R. Bauminger et al., "Iron and reactive oxygen species activity in parkinsonian substantia nigra," Parkinsonism and Related Disorders, vol. 16, no. 5, pp. 329-333, 2010.

[36] R. L. Schroeder and J. P. Gerber, "A reappraisal of Fe(III) adsorption by melanin," Journal of Neural Transmission, vol. 121, no. 12, pp. 1483-1491, 2014.

[37] G. Bartzokis, P. H. Lu, K. Tingus et al., "Gender and iron genes may modify associations between brain iron and memory in healthy aging," Neuropsychopharmacology, vol. 36, no. 7, pp. 1375-1384, 2011.

[38] D. Kaur and J. Andersen, "Does cellular iron dysregulation play a causative role in Parkinson's disease?" Ageing Research Reviews, vol. 3, no. 3, pp. 327-343, 2004.

[39] M. R. Hennis, K. W. Seamans, M. A. Marvin, B. H. Casey, and M. S. Goldberg, "Behavioral and neurotransmitter abnormalities in mice deficient for Parkin, DJ-1 and superoxide dismutase," PLoS ONE, vol. 8, no. 12, Article ID e84894, 2013.

[40] J. Kristinsson, J. Snaedal, G. Tórsdóttir, and T. Jóhannesson, "Ceruloplasmin and iron in Alzheimer's disease and Parkinson's disease: a synopsis of recent studies," Neuropsychiatric Disease and Treatment, vol. 8, pp. 515-521, 2012.

[41] S. A. Schneider, P. Dusek, J. Hardy, A. Westenberger, J. Jankovic, and K. P. Bhatia, "Genetics and pathophysiology of neurodegeneration with brain iron accumulation (NBIA)," Current Neuropharmacology, vol. 11, no. 1, pp. 59-79, 2013.

[42] T. Litwin, G. Gromadzka, G. M. Szpak, K. Jabłonka-Salach, E. Bulska, and A. Członkowska, "Brain metal accumulation in Wilson's disease," Journal of the Neurological Sciences, vol. 329, no. 1-2, pp. 55-58, 2013.

[43] L. Batista-Nascimento, C. Pimentel, R. A. Menezes, and C. Rodrigues-Pousada, "Iron and neurodegeneration: from cellular homeostasis to disease," Oxidative Medicine and Cellular Longevity, vol. 2012, Article ID 128647, 8 pages, 2012.

[44] I. de Domenico, D. M. Ward, M. C. B. di Patti et al., "Ferroxidase activity is required for the stability of cell surface ferroportin in cells expressing GPI-ceruloplasmin," The EMBO Journal, vol. 26, no. 12, pp. 2823-2831, 2007.

[45] K. Kaneko, A. Hineno, K. Yoshida, and S.-I. Ikeda, "Increased vulnerability to rotenone-induced neurotoxicity in ceruloplasmin-deficient mice," Neuroscience Letters, vol. 446, no. 1, pp. 5658, 2008.

[46] S. J. Texel, X. Xu, and Z. L. Harris, "Ceruloplasmin in neurodegenerative diseases," Biochemical Society Transactions, vol. 36, no. 6, pp. 1277-1281, 2008. 


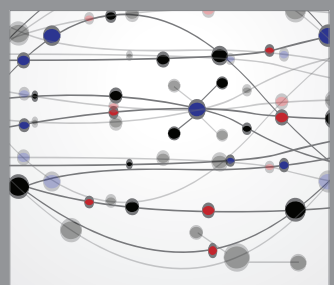

The Scientific World Journal
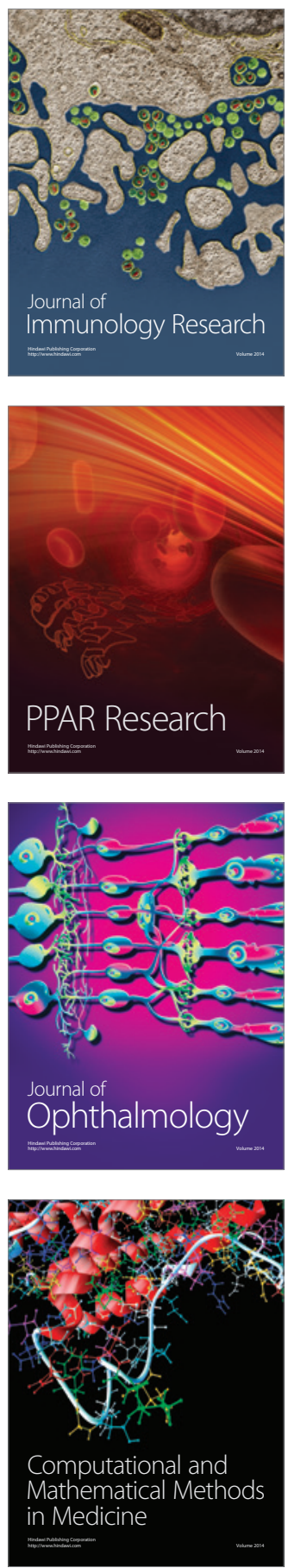

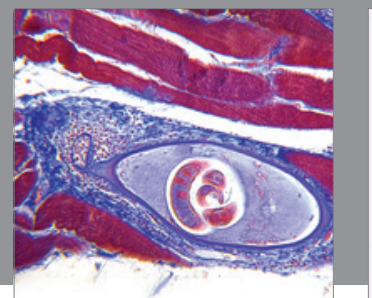

Gastroenterology

Research and Practice
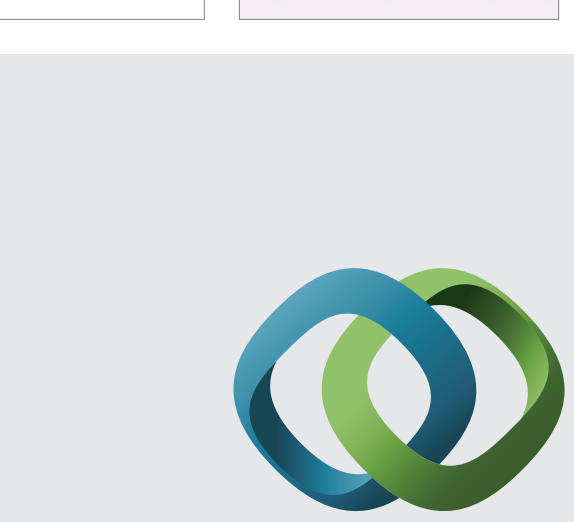

\section{Hindawi}

Submit your manuscripts at

http://www.hindawi.com
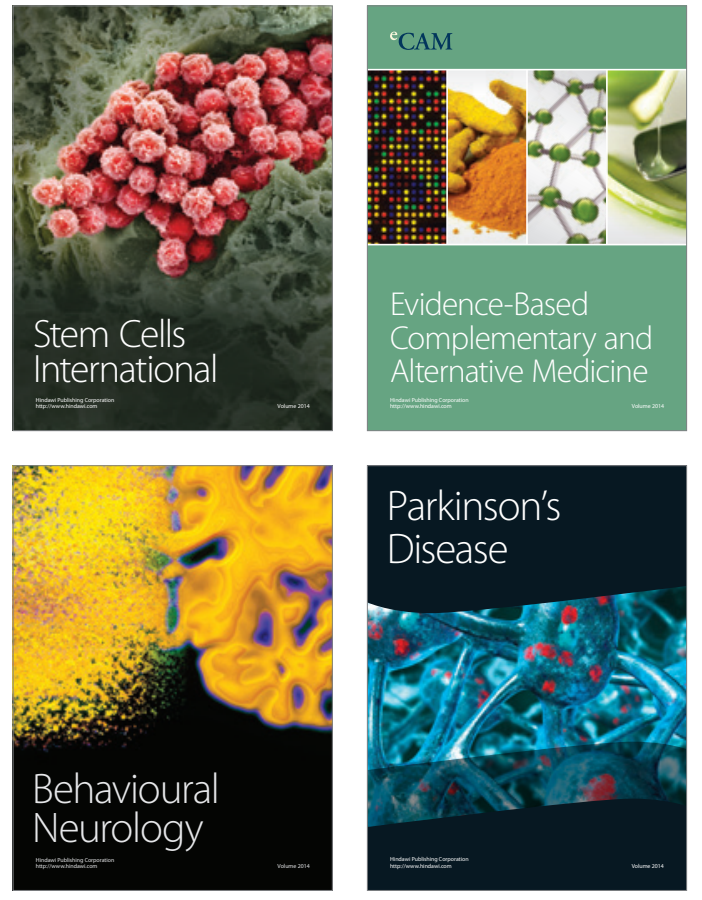
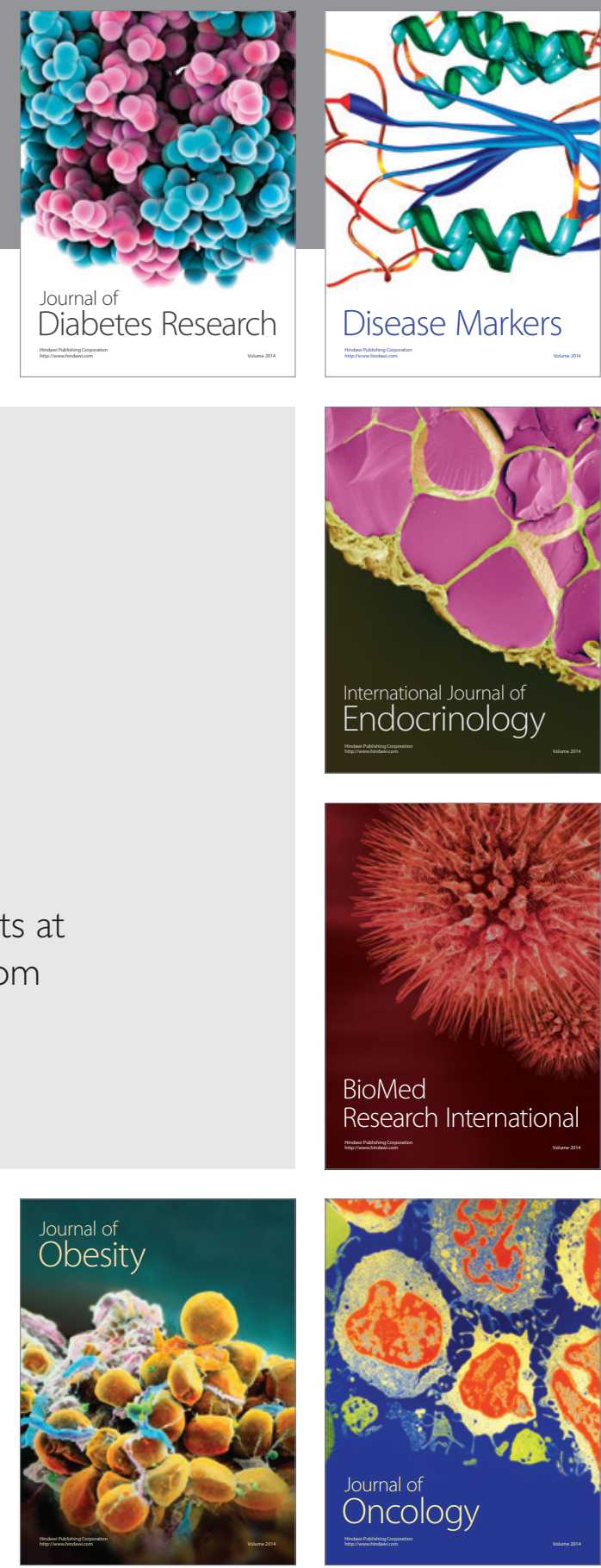

Disease Markers
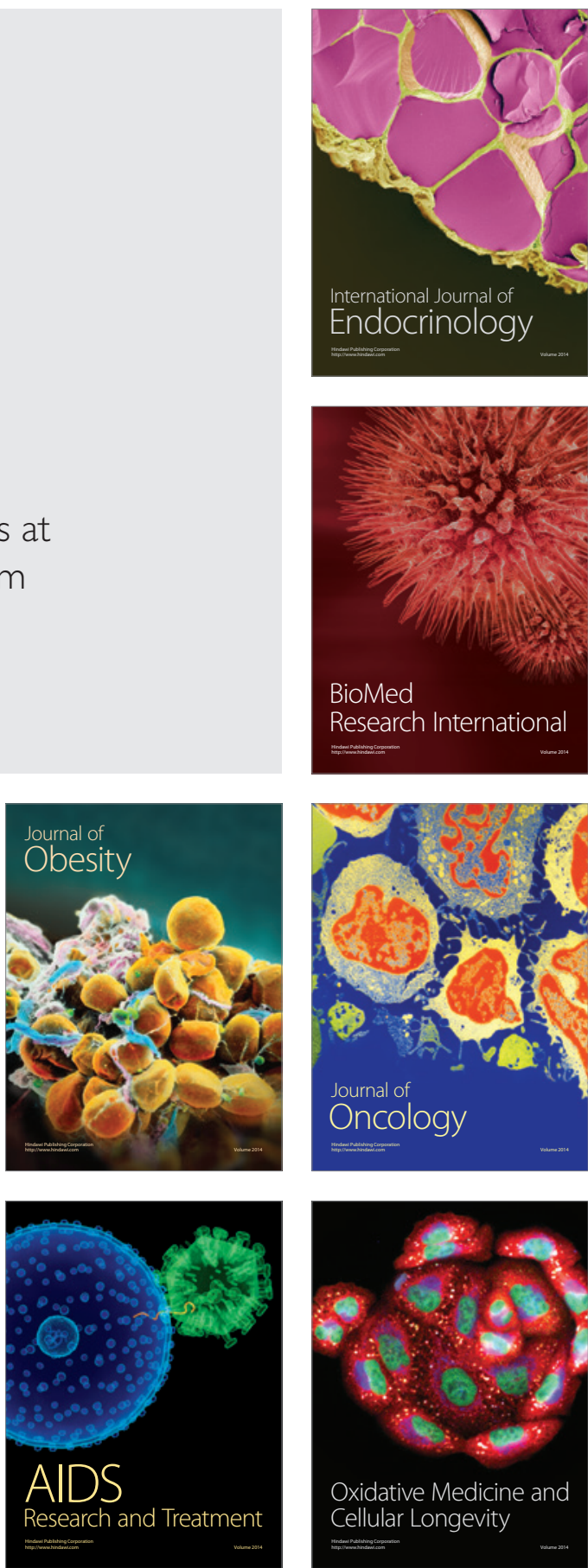